\title{
ZRO Drift Reduction of MEMS Gyroscopes via Internal and Packaging Stress Release
}

\author{
Pengfei Xu ${ }^{1,2}$, Zhenyu Wei ${ }^{1,2}$, Lu Jia ${ }^{1,2}$, Yongmei Zhao', Guowei Han ${ }^{1, *}$, Chaowei Si ${ }^{1, *}$, Jin Ning ${ }^{1,3,4}$ and Fuhua \\ Yang ${ }^{1,2, *}$
}

1 Engineering Research Center for Semiconductor Integrated Technology, Institute of Semiconductors, Chinese Academy of Sciences, Beijing 100083, China; xupengfei@semi.ac.cn (P.X.); zywei97@semi.ac.cn (Z.W.); jialu@semi.ac.cn (L.J.); ymzhao@semi.ac.cn (Y.Z.); ningjin@semi.ac.cn (J.N.)

2 College of Materials Science and Opto-Electronic Technology, University of Chinese Academy of Sciences, Beijing 100049, China

3 School of Electronic, Electrical and Communication Engineering, University of Chinese Academy of Sciences, Beijing 100049, China

4 State Key Laboratory of Transducer Technology, Chinese Academy of Sciences, Beijing 100083, China

* Correspondence: hangw1984@semi.ac.cn (G.H.); schw@semi.ac.cn (C.S.); fhyang@semi.ac.cn (F.Y.); Tel.: +86-10-8230-5403 (G.H.)

\begin{abstract}
Zero-rate output (ZRO) drift induces deteriorated micro-electromechanical system (MEMS) gyroscope performances, severely limiting its practical applications. Hence, it is vital to explore an effective method toward ZRO drift reduction. In this work, we conduct an elaborate investigation on the impacts of the internal and packaging stresses on the ZRO drift at the thermal start-up stage, and propose a temperature-induced stress release method to reduce the duration and magnitude of ZRO drift. Self-developed high-Q dual mass tuning fork gyroscopes (TFGs) are adopted to study the correlations between temperature, frequency and ZRO drift. Furthermore, a rigorous finite element simulation model is built based on the actual device and packaging structure, revealing the temperature and stresses distribution inside TFGs. Meanwhile, the relationship between temperature and stresses are deeply explored. Moreover, we introduce a temperature-induced stress release process to generate thermal stresses and reduce the temperature-related device sensitivity. By this way, the ZRO drift duration is drastically reduced from $\sim 2000 \mathrm{~s}$ to $\sim 890 \mathrm{~s}$, and the drift magnitude decreases from $\sim 0.4 \%$ to $\sim 0.23 \%$. This stress release method achieves a small bias instability (BI) of $7.903 \% \mathrm{~h}$ and a low angle random walk (ARW) of $0.792 \% \mathrm{~V}$, and the longterm bias performance is significantly improved.
\end{abstract}

Keywords: ZRO drift; MEMS gyroscope; internal and packaging stresses; finite element analysis; stress release

\section{Introduction}

Micro-electromechanical system (MEMS) gyroscopes, a kind of Coriolis effect-based inertial sensors, are widely employed in the fields of aerospace, positioning, navigation and consumer electronics [1,2]. With the continuously developing design, manufacture and control techniques of MEMS gyroscopes, their accuracy and precision have gained a constant improvement $[3,4]$. Nowadays, a majority of MEMS gyroscopes work utilizing the capacitive detection, mode-split and phase-sensitive demodulation architecture. Nevertheless, this architecture will undoubtedly introduce a zero-rate output (ZRO) drift effect, severely limiting the gyroscope accuracy $[5,6]$.

Many studies have reported the origin of gyroscope ZRO drift. For one thing, the gyroscope's resonant frequency is susceptive to its generated heats and environmental fluctuations due to its temperature-sensitive inherent frequency. On this occasion, welldesigned materials and structures with opposite temperature-related characteristics are often used for temperature compensation in practical gyroscope manufacturing $[7,8]$. For 
another, the drive frequency can be used as a temperature indicator for the first-order temperature compensation, and thus a sub-degree-per-hour bias stability $(1 \sigma)$ is attained [9]. The inevitable phase delay through the sense model induces partial quadrature fluctuations into the in-phase channel, and this in-phase and quadrature (IQ) coupling will exacerbate the ZRO drift [10]. A one-time phase self-compensation method was reported to eliminate the circuit phase delay, and the temperature sensitivity of ZRO drift was greatly reduced [11]. Qian Shen et al. investigated the bias-temperature relationship during the thermal start-up process, and found that the gyroscope frequency variation is closely related to the heating of the gyroscope and its control circuit operation [12]. Moreover, the adhesion layer between gyroscope and packaging shell may induce the packaging stress when there appears environmental vibration or temperature instability $[13,14]$. Gyroscopes' internal stresses and the packaging stresses may also contribute to the ZRO drift properties, however, no related research work has been reported. It is meaningful to expound the effects of internal and packaging stresses on ZRO drift, and an effective approach is also in great need to reduce the duration and magnitude of ZRO drift.

In this work, we, for the first time, detailly investigate the impacts of the internal and packaging stresses on gyroscopes' ZRO drift, and demonstrate a temperature-induced stress release method to reduce the duration and magnitude of ZRO drift. We adopt selfdeveloped high-Q dual mass tuning fork gyroscopes (TFGs) with dedicated control circuit to explore the correlations between ZRO drift and temperature at the thermal start-up stage. To uncover gyroscope's inside temperature distribution and the temperature-dependent stress variation in its working process, we built a rigorous finite element simulation model based on the actual device and package structure. Moreover, we propose a feasible method for system stress release by cyclically applying high and low temperatures onto devices. With the assistance of generated thermal stresses, the temperature sensitivity of internal and packaging stresses is significantly reduced, effectively reducing the impacts of temperature variation on the ZRO drift during TFGs' thermal start-up processes. Notably, we conduct an in-depth study about the effects of the internal and packaging stresses on the ZRO drift, and report a genuine improvement toward the duration and magnitude of ZRO drift, providing a new promise for improving the long-term bias performances of MEMS gyroscopes.

The rest of this paper is organized as follows. Section 2 gives the structure and packaging of TFG. Section 3 describes the research on the ZRO drift of TFGs. And the numerical simulation of internal and packaging stresses is given in Section 4 . Section 5 presents the temperature-induced stress release process and its impact on the ZRO drift. Finally, Section 6 concludes this paper.

\section{Structure and Packaging of TFG}

To investigate the impact of gyroscopes' internal stresses of gyroscope and the packaging stresses on the ZRO drift at the thermal start-up stage, self-developed high-Q dual mass TFGs, which are fabricated with through-silicon-via (TSV) and glass-in-silicon (GIS) reflow processes [15], is adopted in this paper.

The schematic and scanning electron microscope (SEM) photograph of the self-developed high-Q dual mass TFG is shown in Figure 1. It consists of two symmetricallydecoupled proof masses and two symmetrically-decoupled lever, which ensure the suppression of common modes and the parasitical low-frequency in-phase mode. When the electrostatic excitation is applied in the drive elements, the proof masses begin to vibrate along the drive axis and then maintain a constant amplitude and frequency vibration under the negative feedback control. If there is an angular velocity input in the Z-axis, according to the Coriolis effect [16], the corresponding output will be obtained through the sense elements, which is proportional to the input angular velocity. From the SEM photograph, the surface of the dual mass TFG is flat, besides, the surfaces, comb teethes and sidewalls of the TFG structures are smooth, indicating that the manufactured TFGs have excellent device characteristics. 


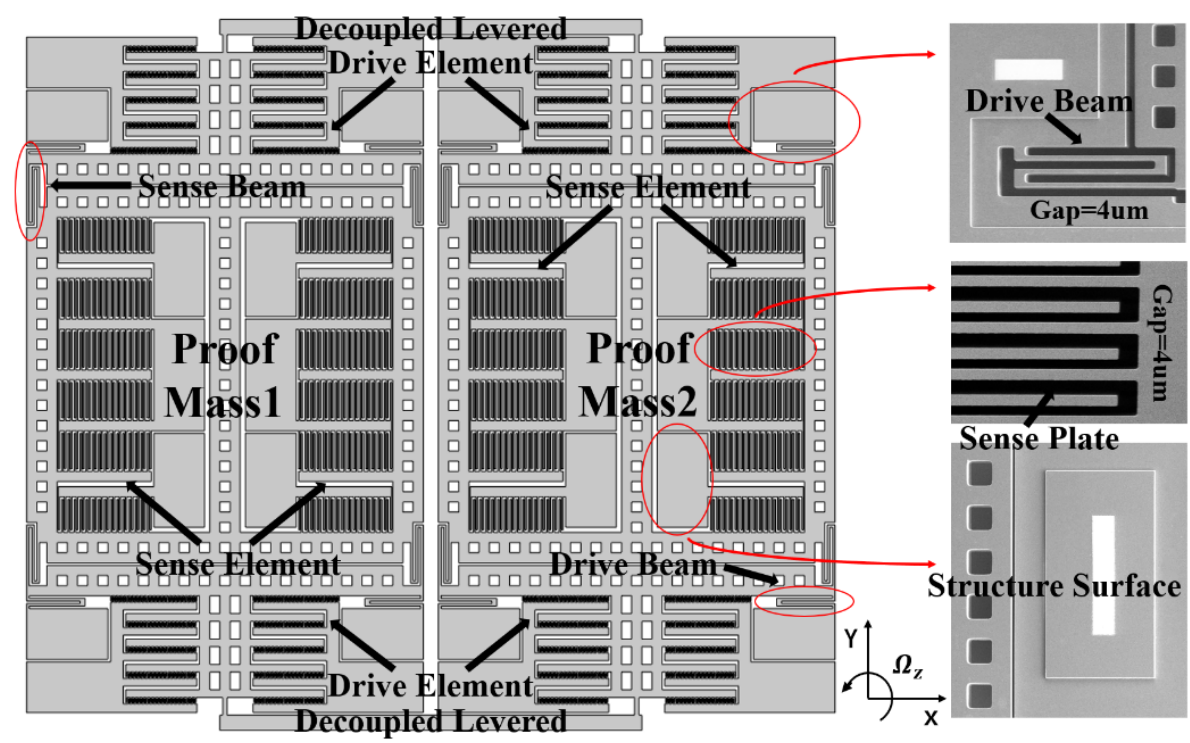

Figure 1. Schematic and SEM photograph of the dual mass TFG.

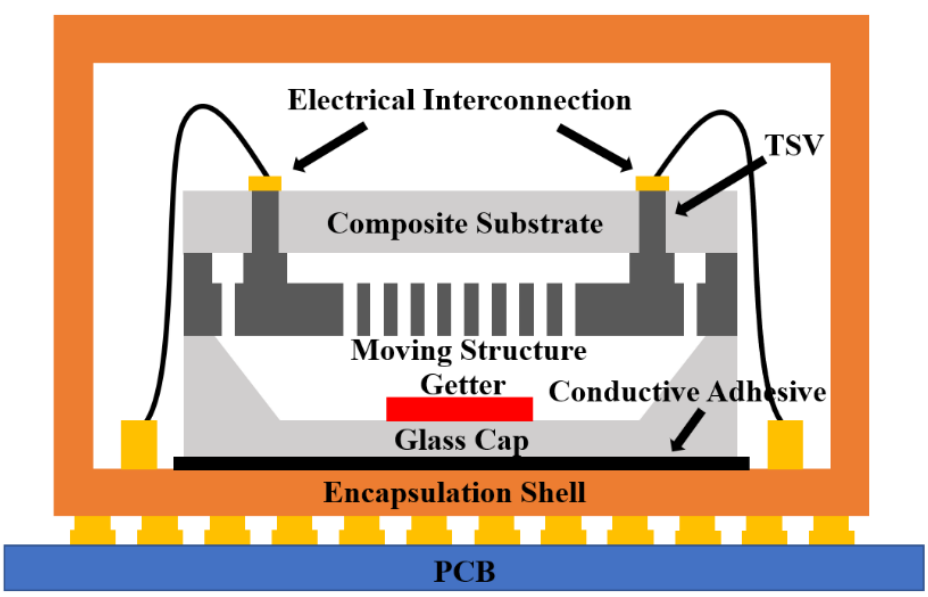

(a)

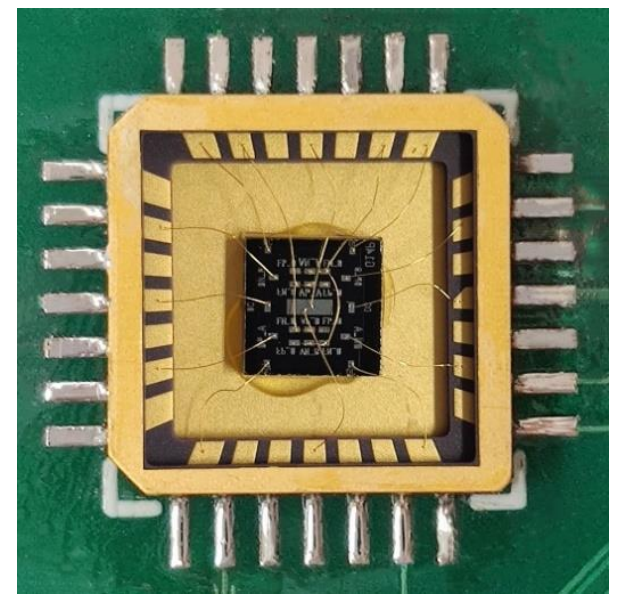

(b)

Figure 2. (a) Side view and (b) Ceramic carrier photograph of the vacuum-packaged MEMS TFG.

As illustrated in Figure 2 (a), the dual mass TFG is composed of the composite substrate, the moving structure and the class cap. Then the class cap of the wafer-level packaged (WLP) TFG is affixed to the encapsulation shell by using a conductive adhesive. The electrical interconnection between the aluminum electrodes and the shell electrodes is realized by wire bonding. Finally, the encapsulation shell and control circuit system are placed on a printed circuit board (PCB) to realize loop control and signal processing. To reduce the stress mismatch between the WLP TFG and the encapsulation shell, a ceramic carrier (CLCC28) is used for its thermal coefficient of expansion (TCE) is close to silicon, and the ceramic carrier photograph is shown in Figure 2 (b).

The block diagram of the control circuit system is depicted in Figure 3, including the front-end analog circuit, signal conversion module and field-programmable gate array (FPGA). The front-end analog circuit is mainly responsible for signal acquisition, capacitor-voltage conversion, signal amplification and filtering. And the capacitor signals of TFG are modulated and demodulated by ring diodes to improve signal-to-noise ratio (SNR). A signal conversion module, which contains two 14 bits analog-to-digital (ADC) converters (ADS5553) and three 14 bits digital-to-analog (DAC) converters (DAC2904), is used for the conversion of analog and digital signals. Then, the acquired signals are transmitted between the FPGA and the conversion module via the serial peripheral interface (SPI) protocol. Moreover, the signal processing and loop control are implemented in the 
FPGA, in which the automatic gain control (AGC) and phase-locked loops (PLLs) are designed in drive mode to keep the vibration amplitude and frequency stable. Both the quadrature error correction and in-phase loops are fully closed-loop to suppress the quadrature error and read the angular velocity signal. Hence, the sense mode of TFG is rebalanced by using electrostatic forces.

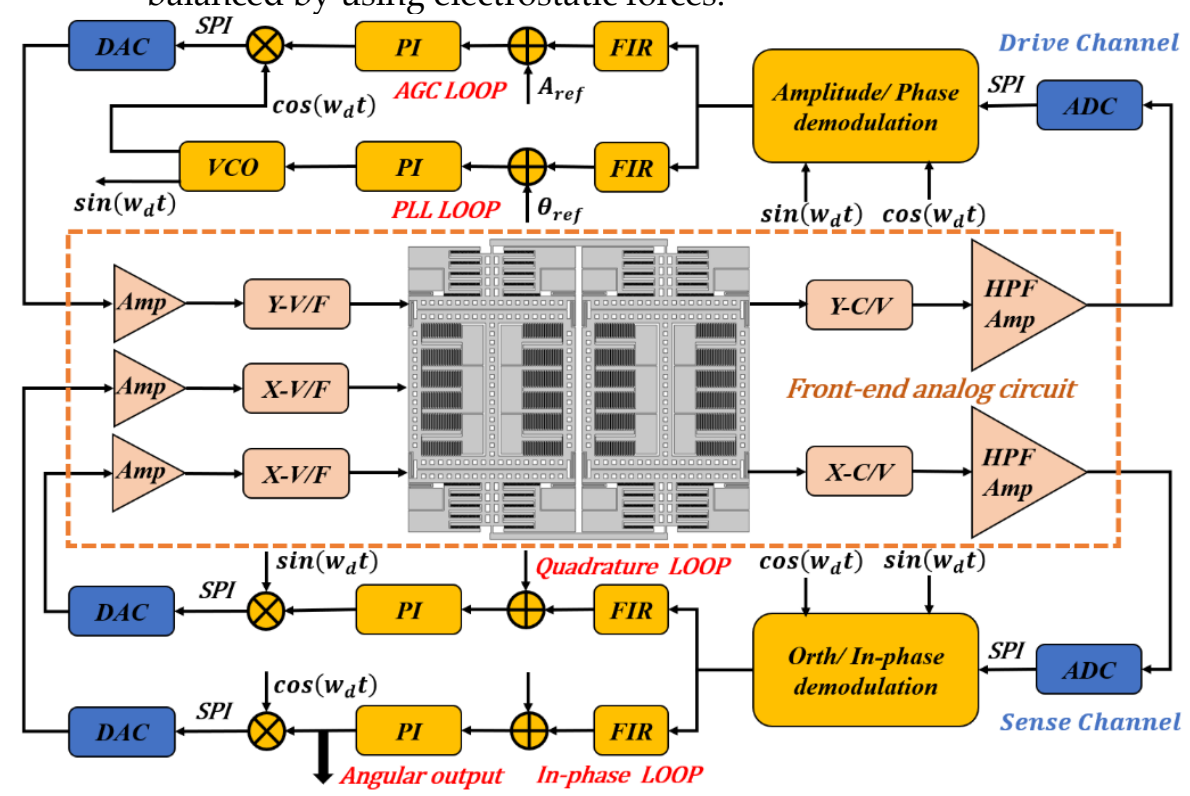

Figure 3. Block diagram of the control circuit system.

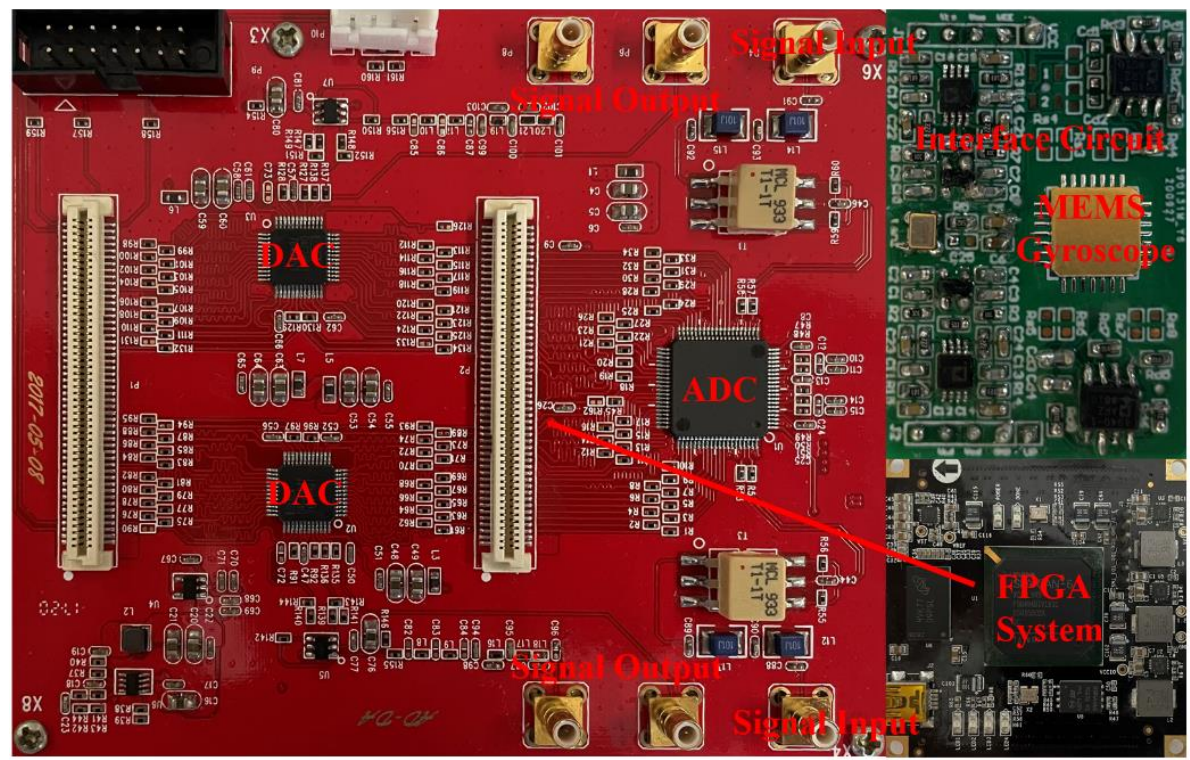

Figure 4. The photograph of the actual test system.

An experimental platform is constructed as described in the previous section and shown in Figure 4. The control loops designed above are cured into the FPGA, and the ceramic carrier is mounted on the interface circuit. As shown in the photograph, it is composed of three parts, and a computer is used for data transfer and real-time loop control with FPGA by universal synchronous asynchronous receiver transmitter (USART).

The TFG and control circuit system is placed on a single-axis rate table (TBL-S1101AT03) to investigate the response to the input angular velocity. Firstly, the control circuit system is powered on, and the angular velocity experiment is conducted after the TFG work stably. The different input angular rate from $-1500 \%$ to $+1500 \%$ is applied, each angular rate input is collected for 2 minutes and the measured average value is taken as the standard value of the sample. Then the standard values corresponding to all the input 
angular velocities are recorded and a linear fit is used to obtain the angular velocity response plot. The measured angular rate response and non-linearity of MEMS TFG is shown in Figure 4, and the full-scale range is over $1500 \%$ with a high-rate resolution to $0.1 \%$. Besides, the scale factor (SF) and non-linearity is $1.413 \mathrm{mV} / \%$ and $420 \mathrm{ppm}$, respectively. The experimental results indicate that the TFG has high resolution, large range and excellent angular velocity response. Proving the designed TFG has excellent characteristics and the control loop can effectively make the mass work in the equilibrium position through the closed-loop force feedback. Hence, its suitable to choose those self-developed high-Q dual mass TFGs to explore the ZRO drift at the thermal start-up stage.

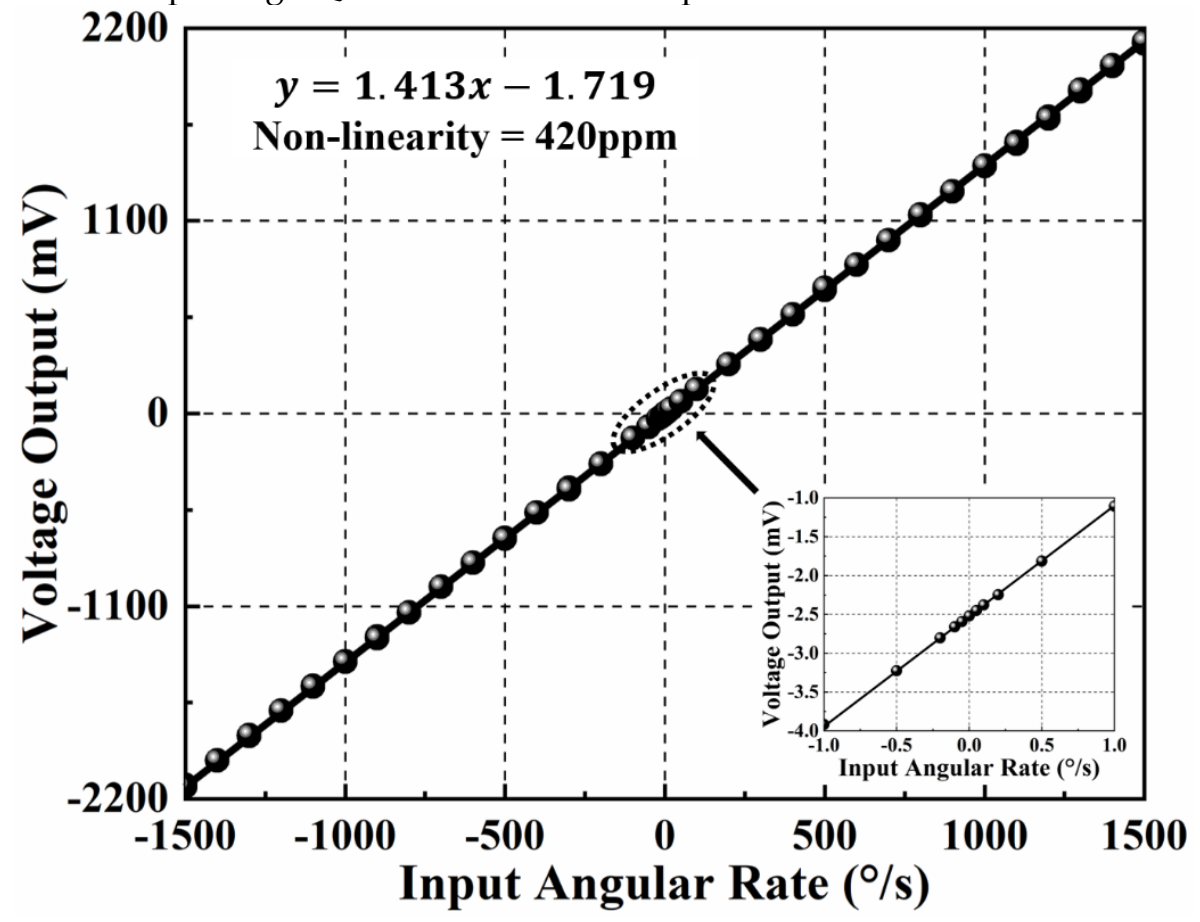

Figure 5. Measured angular rate response and non-linearity of MEMS TFG.

\section{Research on the ZRO Drift of TFGs}

$\mathrm{ZRO}$ is one of the most important performance indicators of MEMS gyroscope, its inevitable drift severely limits the precision and accuracy. Hence, finding an effective method to reduce the ZRO drift is meaningful. To deeply study the output characteristics of the TFGs, four samples are selected, which have similar drive and sense frequencies to ensure the accuracy and reliability of the test. For ZRO measurement, the output angular rate is recorded at $1 \mathrm{kHz}$ sample rate for $4000 \mathrm{~s}$ at room temperature. Basing on the angular rate response and the zero angular velocity output of four samples, the ZRO comparison of four samples is illustrated in Figure 6. It can be seen that the ZRO of four samples shows an obvious drift tendency with the operating time increases. As time goes on, the rate of ZRO drift gradually becomes slower and eventually reach a relatively stable state after a certain time. The duration and magnitudes of ZRO drift for four samples are shown in Table 1. The ZRO drift duration is $\sim 2000 \mathrm{~s}$, and the magnitude of ZRO drift is approximately $0.4 \%$. ZRO drift of gyroscopes directly leads to the reduction of the accuracy of phase sensitive demodulation, and it has a serious impact on the performance of BI, ARW and so on. Hence, it is essential to study the ZRO drift, understand the drift mechanism and find an effective method to shorten the duration and magnitude of $\mathrm{ZRO}$ drift. 


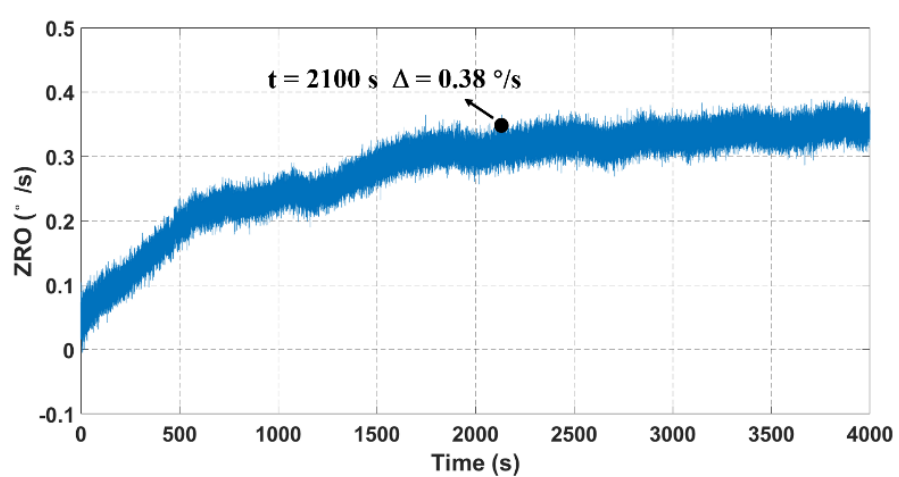

(a)

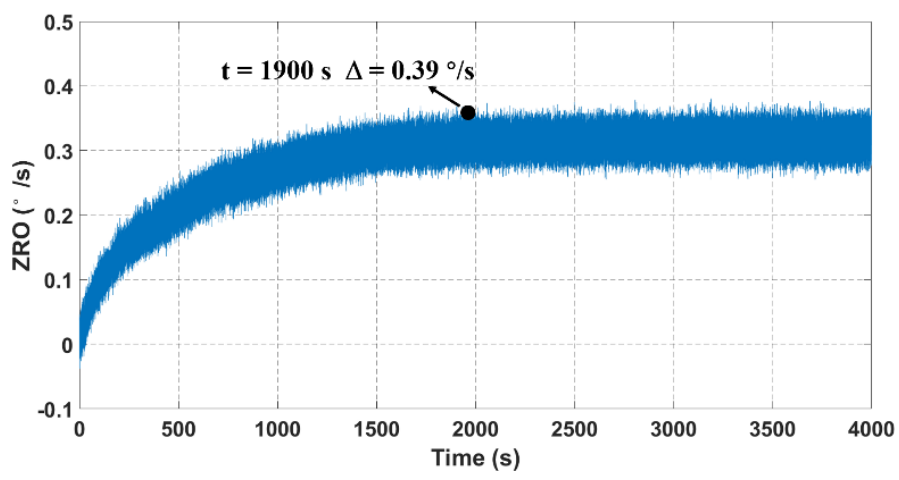

(c)

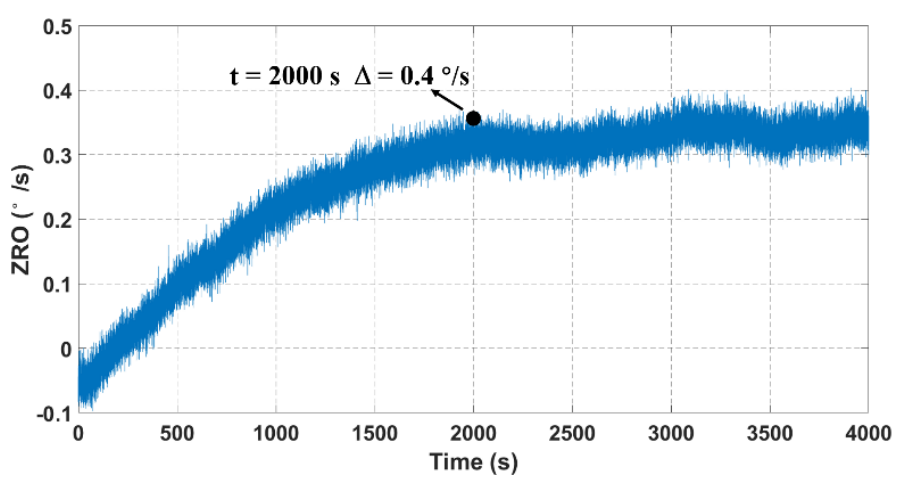

(b)

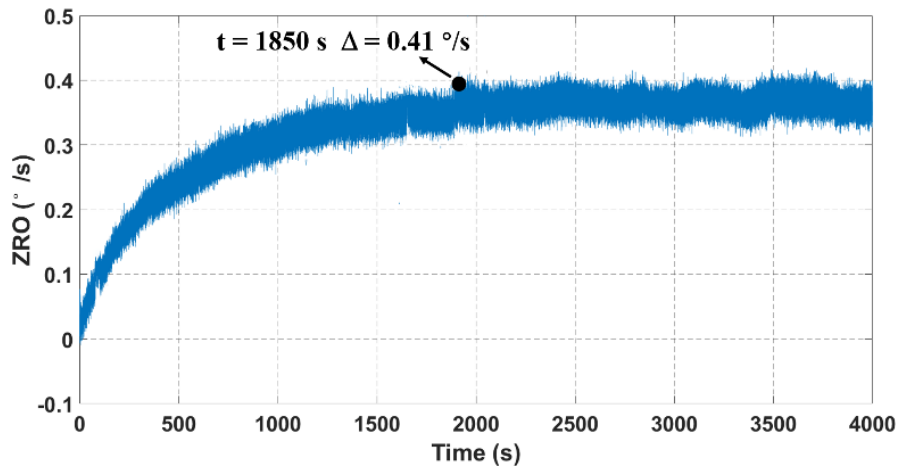

(d)

Figure 6. ZRO comparison at $1 \mathrm{kHz}$ sample rate of four samples.

Table 1. The duration and magnitudes of ZRO drift for four samples

\begin{tabular}{ccccc}
\hline & Sample1 & Sample2 & Sample3 & Sample4 \\
\hline fdrive $\mathbf{( H z )}$ & 11713.5 & 11856 & 11800 & 11515 \\
fsense $^{(H z)}$ & 11612 & 11757.7 & 11696 & 11422.6 \\
Duration (s) & 2100 & 2000 & 1900 & 1850 \\
Magnitude (\%/s) & 0.38 & 0.4 & 0.39 & 0.41 \\
\hline
\end{tabular}

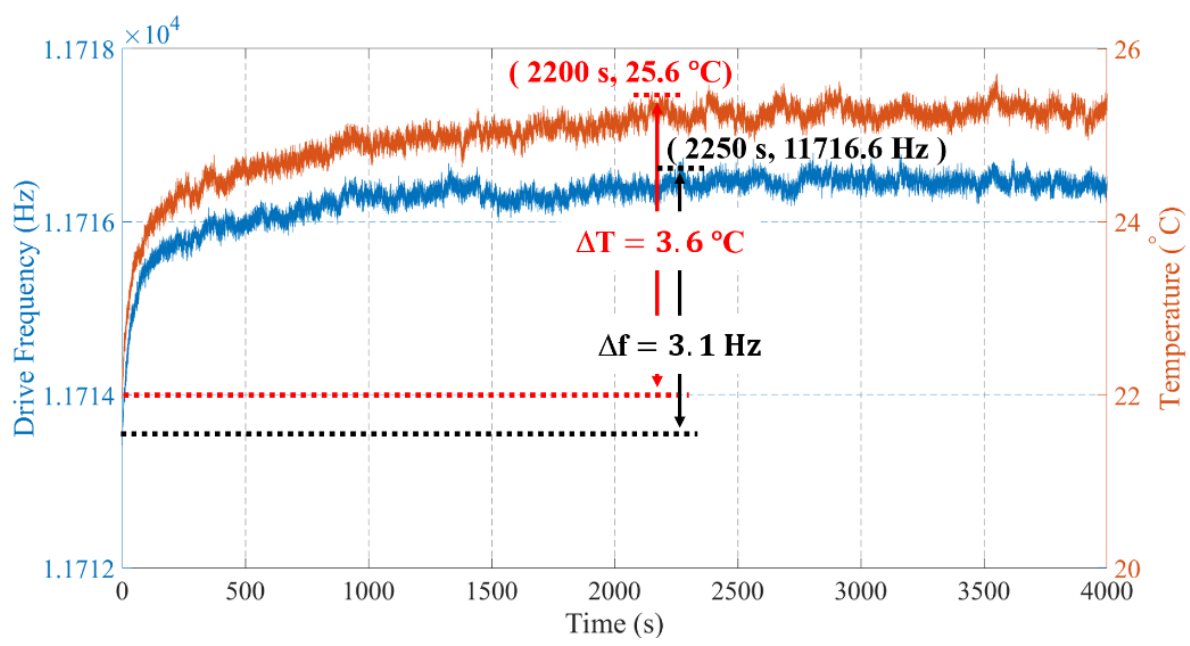

Figure 7. Measured device temperature and drive frequency ( $\left.f_{\text {drive }}\right)$ of sample1.

Research on the mechanism of ZRO drift has been conducted for many years, the $\mathrm{ZRO}$ drift of gyroscopes mainly sources from its inherent sensitivity to temperature variations $[17,18]$. To investigate the correlation between temperature and ZRO drift, a detailed measurement of the device temperature and drive frequency (fdrive) at the thermal stage is adopted. As shown in Figure 7, the device temperature of TFG rises rapidly within $300 \mathrm{~s}$ since the power on, and the rate of increase gradually slows down, reaching a peak 
temperature of $25.6 \%$ at $\sim 2200 \mathrm{~s}$ and tendency to stabilization, the increases in temperature is $\sim 3.6{ }^{\circ} \mathrm{C}$. Besides, the drive frequency (fdrive) has a similar variation to the device temperature, which tends to be stable after $\sim 2250 \mathrm{~s}$ and the variation of drive frequency (fdrive) is $\sim 3.1 \mathrm{~Hz}$. The test results indicate that the ZRO drift has a strong correction with the drive frequency $\left(f_{\text {drive }}\right)$ and device temperature.

One reason for this phenomenon is that the device parameters such as Young's modulus of the gyroscope are sensitive to temperature, causing the frequency of TFG change with temperature variation [19]. Therefore, the drive frequency (fdrive) is often used as one of the compensation indicators of the ZRO drift of gyroscope, many relevant studies focus on this point. On the other hand, the gyroscope and interface circuits will change the temperature of TFGs in its working process. And the mismatches of the TCEs between the gyroscope itself and the package material lends to packaging stress. This packaging stress not only exists between the gyroscope and the package material, but exists inside the gyroscope, causing a significant impact on the ZRO drift of gyroscope.

\section{Numerical Simulation of Internal and Packaging Stresses}

To investigate the impact of internal and packaging stresses on the ZRO drift of MEMS gyroscope, the structure and packaging of TFG is modelled with COMSOL Multiphysics software. As illustrated in the photograph of TFG control circuit (Figure 4). To avoid the adverse effect of heat and thermal stress on the TFG when the components of the control circuit is working, the PCB was designed to allow sufficient space between the gyroscope and the circuit components. Besides, the FPGA module, which generates the most heat, is designed on an additional layer of the PCB. Therefore, we can basically exclude the influence of the circuit components working heat on the gyroscope, and only the gyroscope device itself and the package will be considered in the following simulation analysis.

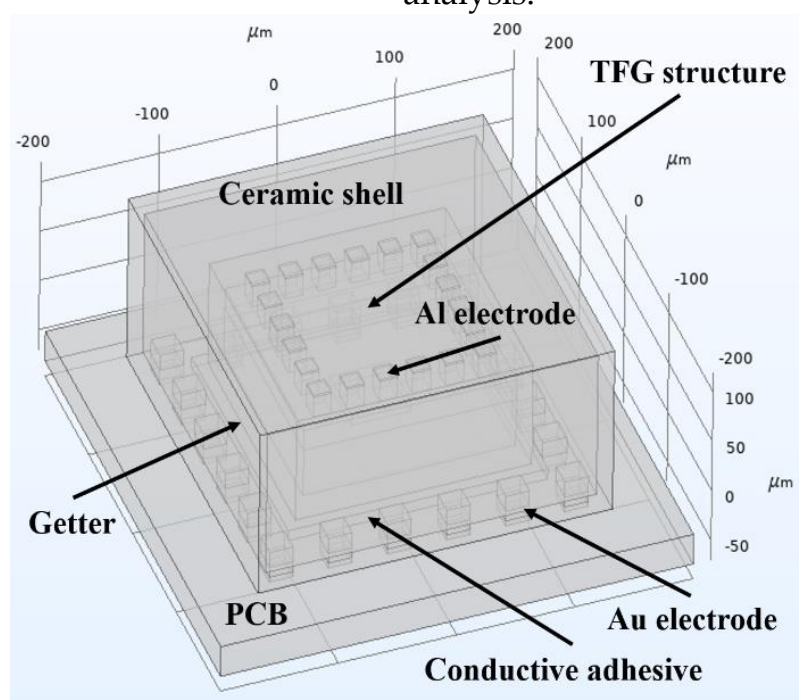

(a)

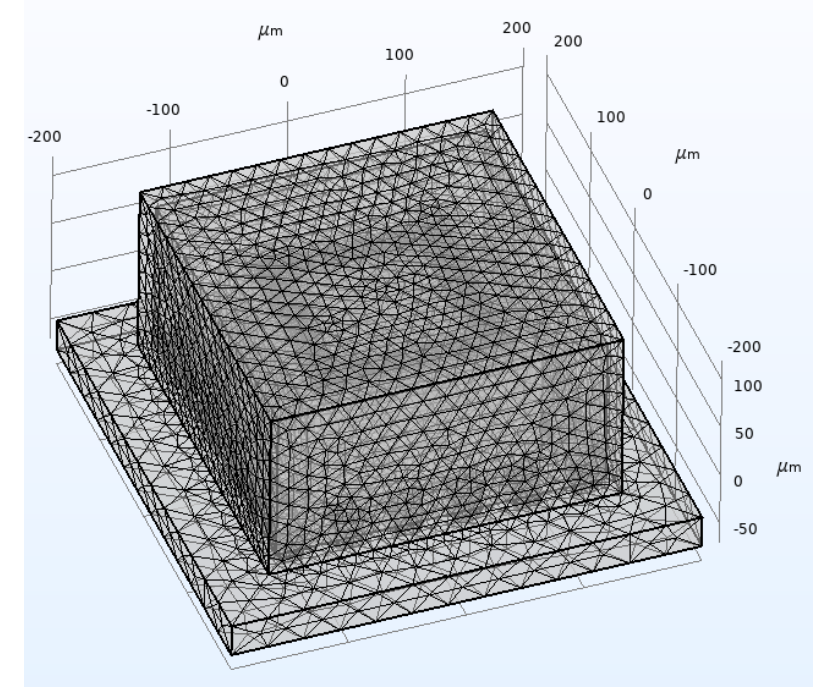

(b)

Figure 8. (a) Simulation model and (b) Grid model of the structures and packaging.

As shown in Figure 8 (a), a rigorous finite element simulation model is built based on the actual device and package structure according to the structure shown in Figure 2 (a) to ensure that it matches the actual situation. Which consists of TFG structure, glass cap, ceramic shell, electrode, PCB and other parts, and the solid mechanics and heat transfer in solids modules with a thermal expansion multi-physics fields are used to study the temperature and stress changes in TFG's working process. The dimensions and essential material properties of components in the simulation model are listed in Table 2, where the thermal conductivity $\kappa$ is the ability of a material to conduct heat directly and the TCE is the volume change for a unit temperature change. The grid model of the TFG structures and packaging system is shown in Figure 8 (b), to get more accurate simulation results, the meshes are divided separately according to the structure and size of different regions. 
Table 2. Dimensions and essential material properties of components in the simulation model.

\begin{tabular}{ccccc}
\hline Components & Dimension (um) & Material & TCE (1/k) & $\mathbf{\kappa}(\mathbf{W} /(\mathbf{m} * \mathbf{k}))$ \\
\hline Ceramic shell & $280^{*} 280^{*} 140$ & $\mathrm{Al}_{2} \mathrm{O}_{3}$ & $6.5 \mathrm{e}-6$ & 35 \\
Glass cap & $200^{*} 200^{*} 100$ & $\mathrm{SiO}_{2}$ & $0.5 \mathrm{e}-6$ & 1.4 \\
TFG structure & $180^{*} 180^{*} 20$ & Silicon & $2.5 \mathrm{e}-6$ & 0.21 \\
Al electrode & $15^{*} 15^{*} 22$ & Aluminum & $23.1 \mathrm{e}-6$ & 237 \\
Au electrode & $20^{*} 20^{*} 30$ & Gold & $14.2 \mathrm{e}-6$ & 317 \\
Getter & $50^{*} 50^{*} 10$ & Titanium & $8.6 \mathrm{e}-6$ & 21.9 \\
PCB & $400^{*} 400^{*} 30$ & Polyimide & $12.3 \mathrm{e}-6$ & 0.15 \\
Conductive adhesive & $220^{*} 220^{*} 10$ & Polyethylene & $150 \mathrm{e}-6$ & 0.38 \\
\hline
\end{tabular}

When setting the boundary conditions and initial conditions of the simulation model, the environment temperature is set at $295.15 \mathrm{k}$ basing on the actual temperature. Since the influence of the circuit system is not considered, the TFG structure is set as the exclusive heat source of the simulation model. The transient temperature distribution of the TFG structures and packaging system is shown in Figure 9 (a). To maintain the ultra-high $Q$ value of the TFG, the cavity is retained at a pressure of $\sim 10^{-5} \mathrm{mbar}$ in the manufacturing process. Hence, the heat conduction of the cavity air can be basically ignored. The heat generated by TFG is conducted to the ceramic shell through the conductive adhesive and electrodes, and eventually most of them is dissipated into the air. Besides, a portion of the heat is conducted to the top of the PCB through the contact between the bottom of ceramic shell and PCB. Eventually, the heat generated in the working process of TFG and the heat dissipation of the system reach a balance, the temperature and the drive frequency ( $f_{\text {drive }}$ ) of the TFG tend to be stable.

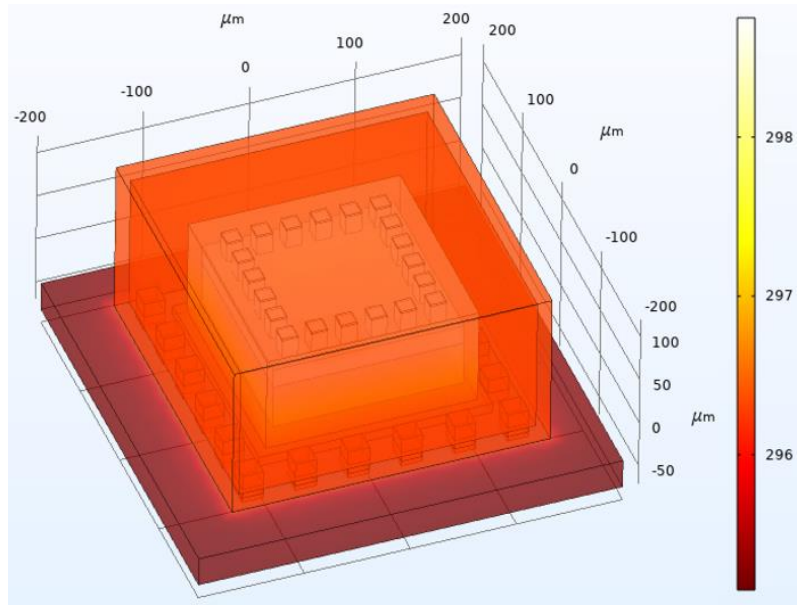

(a)

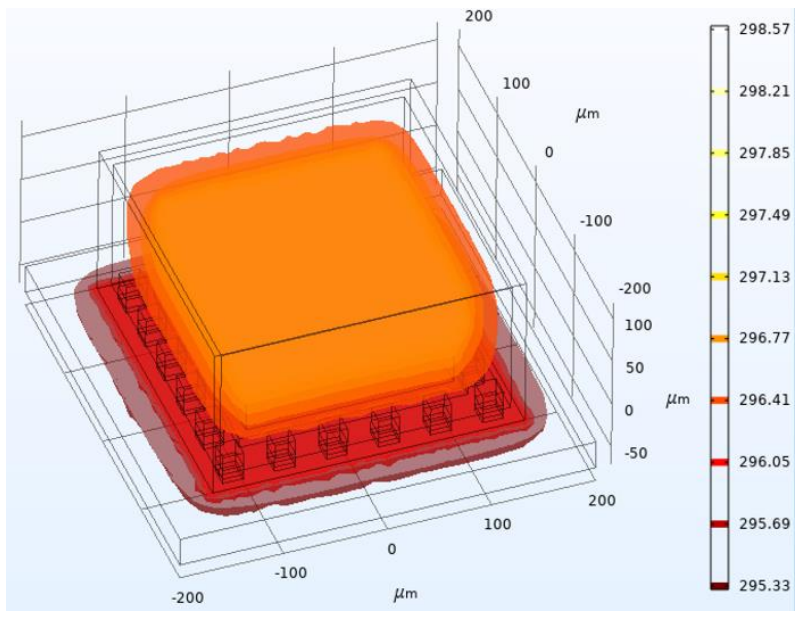

(b)

Figure 9. (a) Transient temperature distribution and (b) isotherm in the working process of TFG.

The isotherm in the working process of TFG is shown in Figure 9 (b), it can be observed that the TFG maintain a relatively high temperature inside the device. Due to the mismatch of materials or the residual stresses from the manufacturing process, the generated heat may cause large strains inside the TFG. Which seriously affects the performance, such as ZRO drift, and even causes the failure of TFG. Therefore, it is essential to deeply investigate the stress distribution inside the device and between the device and packaging in the working process of TFG. 


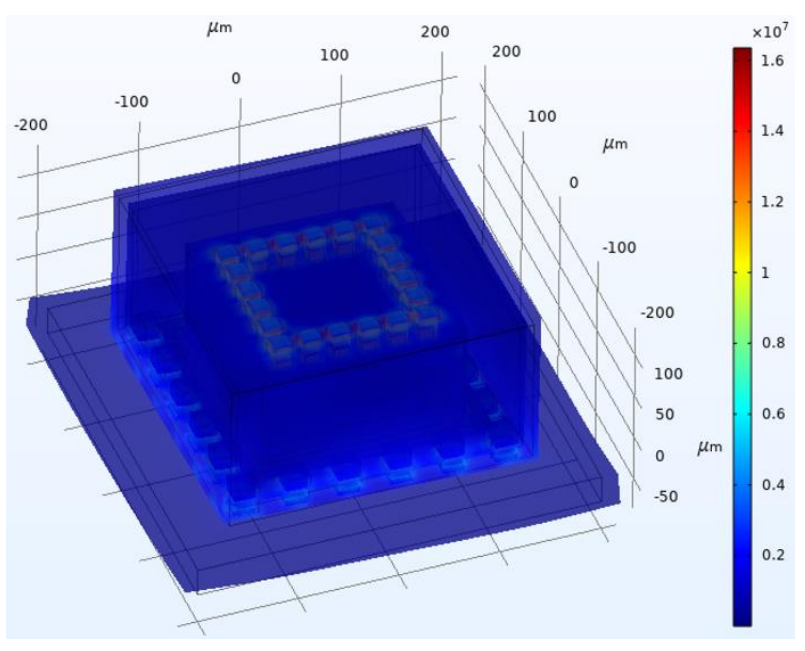

(a)

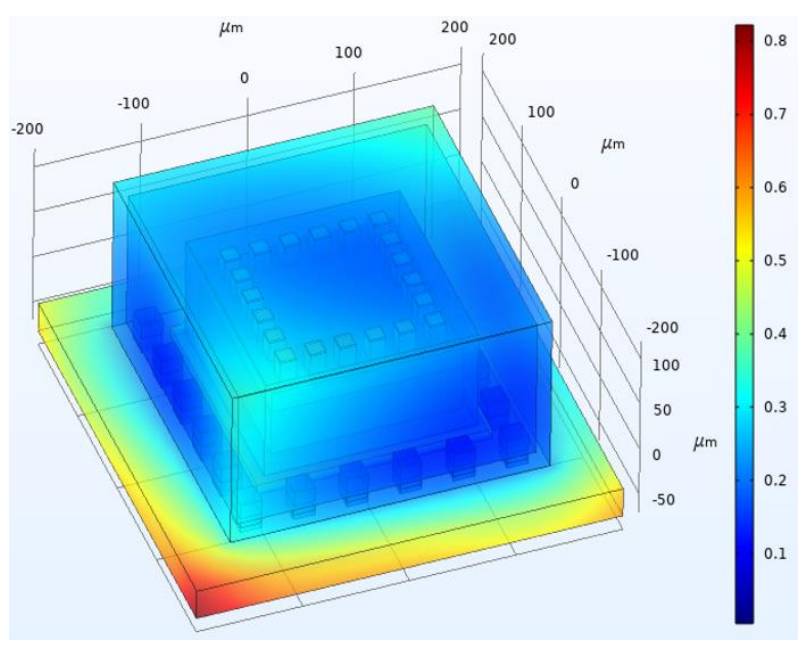

(b)

Figure 10. The stress distribution (a) and (b) displacement at temperature equilibrium of TFG.

As depicted in Figure 10 (a), the heat-induced stress is $\sim 10 \mathrm{MPa}$ in the working process of TFG. The heat-induced stresses are mainly concentrated on the electrodes and the contact surfaces between the TFG and the packaging, and a large warpage exists around the edges of the PCB. Hence, the heat-induced stress may cause severe effects on the TFG internals and the contact surfaces. A similar situation can be observed in Figure 10 (b). Due to the significant TCE differences between TFG and packaging materials, the heatinduced stresses induce significant extrusion and stretching of the interface between the TFG and the package in the working process. The existence of this strain has a potential impact on the performance of TFG, which is particularly noticeable in the frequency.

The packaging of this TFG consists of two main parts, one is the WLP, which provides the necessary vacuum and protection for the sensing structure through an anodic bonding process. Another is the chip-level packaging of wire-bonding, die-bonding, plastic molding and board level interconnection [20]. Moreover, the main sources of the internal stresses are the thermomechanical stresses generated by the bonded wafer and the TCE mismatch among the silicon and glass. Besides, the packaging stresses are mainly caused by the TCE mismatch between TFG and the packaging materials, and the TCE mismatch between the various materials of the package is also an important source of stresses [21]. In particular, the internal and packaging stresses will cause deformation of the TFG structure, leading to frequency change or the damage of TFG.

Therefore, finding a method to relieve the internal and packaging stresses can effectively reduce the impact of heat generated by TFG on the ZRO drift. And the duration and magnitude of ZRO drift can be effectively reduced, which plays a crucial role in enhancing the performance of TFG.

\section{Temperature-Induced Stress Release Experiment.}

High and low temperature cycle is a common test method, often used for failure analysis of MEMS gyroscope. It generates positive and shear stresses between the layers (such as the silicon-glass interface) of gyroscope through temperature-induced stresses. When the high- and low-temperature cycles is applied on the interfaces, the environmental stresses drive the potential defects to crack at the interface due to fatigue, which leads to degradation of the gyroscope performance and may eventually cause the failure of the gyroscope. As the high- and low-temperature shock can be applied to the stress release of the system, the temperature-induced stress release experiment is used to quickly apply high and low temperatures to the samples. The effect of internal and packaging stresses on the ZRO drift is explored by accelerating the stress release of the system.

The four samples are placed in the temperature chamber (TPSTY) for the temperature-induced stress release experiment. The minimum temperature is set as $-20^{\circ} \mathrm{C}$ and the maximum temperature is $70^{\circ} \mathrm{C}$ with $10^{\circ} \mathrm{C} / \mathrm{min}$ temperature change rate. Besides, each 
peak temperature sustains for $10 \mathrm{~min}$, this temperature cycling experiment lasts for 40 cycles to sufficiently release the stresses and the duration time of this is experiment about $26 \mathrm{~h}$.

The temperature-induced stress release experiment generates high and low temperature thermo-induced stress by applying high- and low-temperature cycles. Therefore, the residual mechanical stresses of wafer bonding, the interlayer stresses of $\mathrm{SiO}_{2}$ and $\mathrm{Si}$, and the packaging stresses caused by wiring, molding, and mounting are dramatically released.

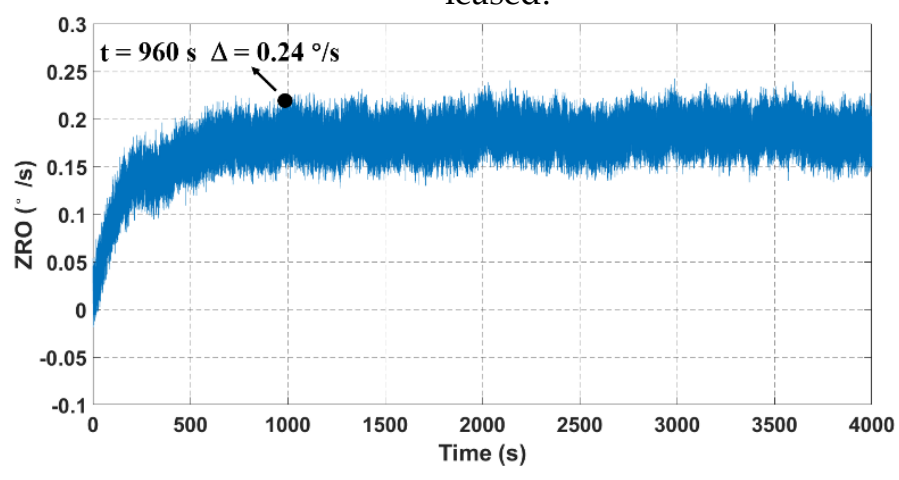

(a)

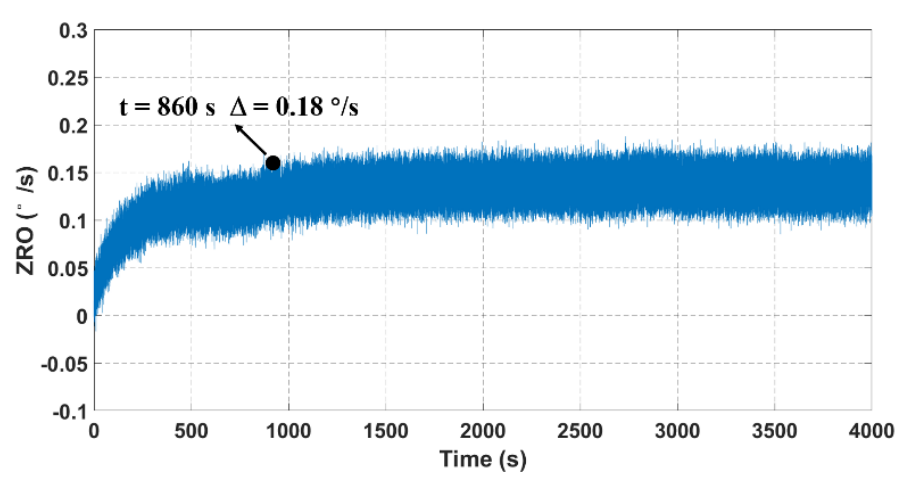

(c)

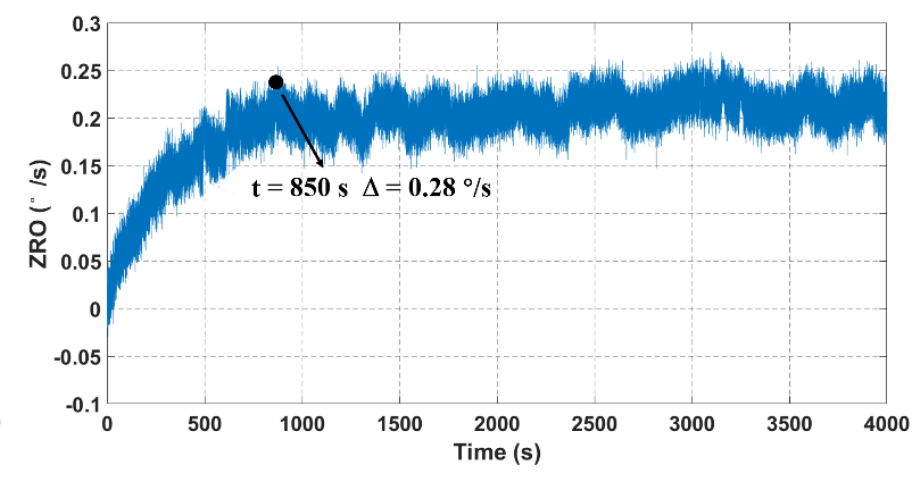

(b)

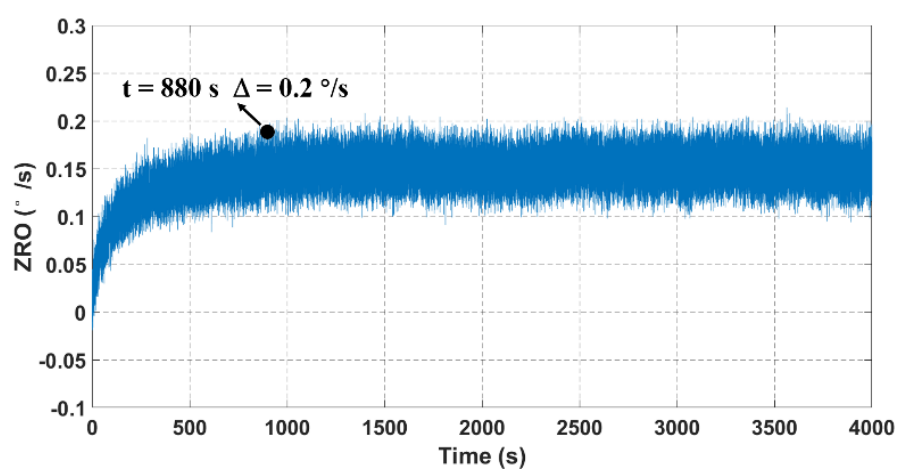

(d)

Figure 11. ZRO comparison after stress release experiment.

Table 3. The duration and magnitudes of ZRO drift after stress release experiment.

\begin{tabular}{ccccc}
\hline & Sample1 & Sample2 & Sample3 & Sample4 \\
\hline Duration (s) & 960 & 850 & 860 & 880 \\
Improve & 2.19 & 2.35 & 2.21 & 2.10 \\
Magnitude (\%) & 0.24 & 0.28 & 0.18 & 0.2 \\
Improve & 1.58 & 1.43 & 2.17 & 2.05 \\
\hline
\end{tabular}

To verify the effectiveness of temperature-induced stress release experiment, ZRO measurements are conducted on the samples after stress release, and the experimental conditions are identical as before. As depicted in Figure 11, the ZRO has a large drift trend at the beginning, gradually slows down and finally stabilizes at the thermal start-up stage. The trend of ZRO drift is approximately the same as before. However, it can be obviously observed that the duration and magnitudes of ZRO drift for four samples have been substantially reduced after temperature-induced stress release experiment. The duration and magnitudes of ZRO drift after stress release experiment is listed in Table 1. It can be seen that the ZRO drift duration is $\sim 890 \mathrm{~s}$, and the magnitude of ZRO drift is approximately $0.23 \%$, which have a substantial improvement. 


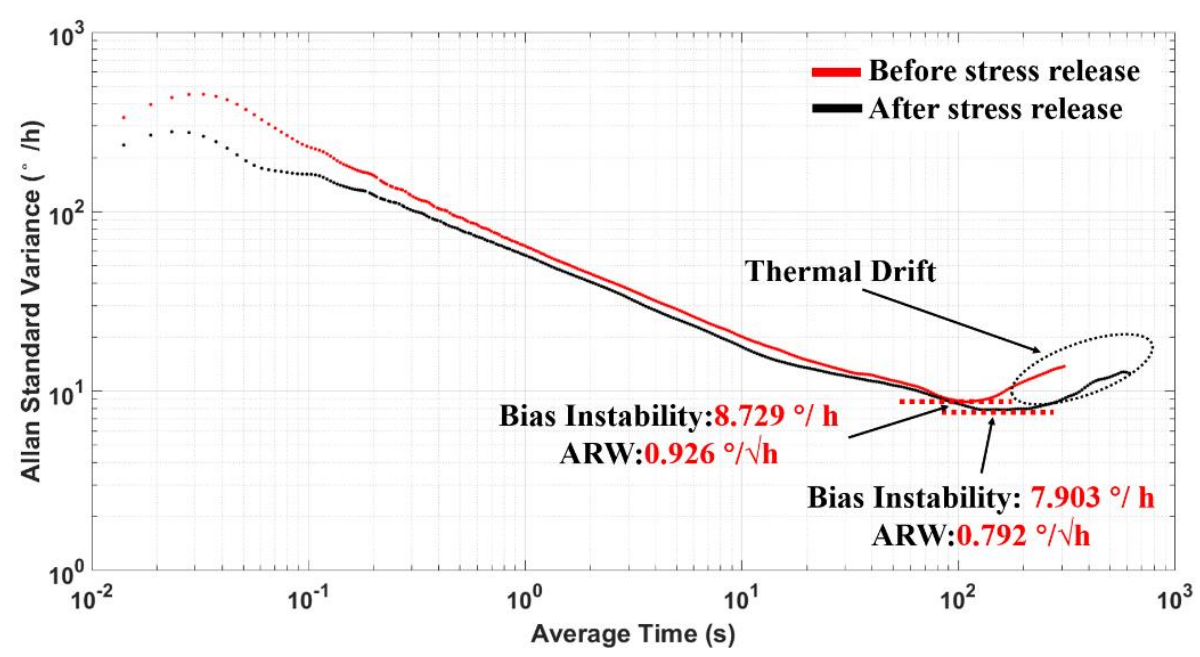

Figure 12. Allan standard variance plot.

To fully investigate the impact of temperature-induced stress release experiment on the performance of TFG, the ZRO data collected above are used. And the Allan variance plot is obtained from the same data set and depicted in Figure 12. It shows that the measured bias instability (BI) is $8.729 \% \mathrm{~h}$ with a $0.926 \% \mathrm{~V} \mathrm{~h}$ angle random walk (ARW) before stress release. Besides, the $\mathrm{BI}$ is $7.903 \% \mathrm{~h}$ and the ARW is $0.792 \% \mathrm{~V}$ h after stress release. A significant reduction in the thermal drift can be observed after temperature-induced stress release experiment, it indicates that the ZRO drift can be effectively reduced.

By cyclically applying high and low temperatures, the sensitivity of internal and packaging stresses to the change of temperature is greatly reduced, which significantly alleviates the impact of temperature change on frequency caused by stresses in the working process of TFG. The experimental results demonstrate that the proposed temperatureinduced stress release experiment can effectively reduce the impact of internal and packaging stresses on the ZRO drift and effectively improve the long-term bias performance of TFGs.

\section{Conclusions}

In summary, we investigate the impacts of internal and packaging stresses of gyroscopes on their ZRO drift properties at the thermal start-up stage. Based on self-developed high-Q dual mass TFGs, it is discovered that the gyroscope drive frequency badly drifts during its normal operation, due to the heat and also the stress-induced effects. Correspondingly, the ZRO drift is non-negligible in this situation. With a finite element simulation, the inside temperature distribution together with the relationship between temperature and stresses is further revealed for our TFGs. Besides, we demonstrate a temperature-induced stress release experiment to generate thermal stresses to the TFG and packaging. By cyclically applying high and low temperatures, the temperature-related device sensitivity about stresses is restrained. Thus, the ZRO drift duration and magnitude are drastically reduced by a factor of $\sim 1.7$ and $\sim 2.2$, respectively. And a significant reduction in the thermal drift can be observed, meaning an improved long-term bias performance of TFGs. Moreover, a small low BI of $7.903 \% \mathrm{~h}$, and a small ARW of $0.792 \% \sqrt{ } \mathrm{h}$ are realized. This work provides a new thought to improve the performances of MEMS gyroscopes, bringing a new promise for their practical applications.

Author Contributions: Conceptualization, Pengfei Xu; Data curation, Pengfei $\mathrm{Xu}$, Zhenyu Wei and Fuhua Yang; Resources, Guowei Han, Chaowei Si and Jin Ning; Software, Pengfei Xu, Yongmei Zhao; Supervision, Guowei Han, Chaowei Si, Jin Ning and Fuhua Yang; Validation, Pengfei Xu and Lu Jia; Writing - original draft, Pengfei Xu; Writing - review \& editing, Guowei Han, Chaowei Si, Jin Ning and Fuhua Yang. 
Funding: This work is supported by the National Key R\&D Program of China (No.2018YFF01010500) and Chinese National Science Foundation (Contract No. 61974136 and No. 52075519).

Data Availability Statement: Not applicable

Acknowledgments: Not applicable

Conflicts of Interest: The authors declare no conflict of interest.

\section{Appendix A}

The appendix is an optional section that can contain details and data supplemental to the main text-for example, explanations of experimental details that would disrupt the flow of the main text but nonetheless remain crucial to understanding and reproducing the research shown; figures of replicates for experiments of which representative data is shown in the main text can be added here if brief, or as Supplementary data. Mathematical proofs of results not central to the paper can be added as an appendix.

\section{Appendix B}

All appendix sections must be cited in the main text. In the appendices, Figures, Tables, etc. should be labeled starting with "A" - e.g., Figure A1, Figure A2, etc.

\section{References}

[1] Fang, Y.; Fu, W.; An, C.; Yuan, Z.; Fei, J. Modelling, Simulation and Dynamic Sliding Mode Control of a MEMS Gyroscope[J]. Micromachines, 2021, 12(2):190.

[2] Marx, M.; D.D, Dorigo.; Nessler, S.; Rombach, S.; Manoli, Y. 9.4 A 27 $\mu W$ 0.06mm 2 background resonance frequency tuning circuit based on noise observation for a $1.71 \mathrm{~mW}$ CT- $\Delta \Sigma$ MEMS gyroscope readout system with $0.9^{\circ} / \mathrm{h}$ bias instability[C]// Solid-state Circuits Conference. IEEE, 2017.

[3] Rafael, V.; Raquel, V.; Felipe, M. A Low Cost Sensors Approach for Accurate Vehicle Localization and Autonomous Driving Application[J]. Sensors, 2017, 17(10):2359-.

[4] Xu, P.; Wei, Z.; Guo, Z.; Jia, L.; Yang, F. A Real-Time Circuit Phase Delay Correction System for MEMS Vibratory Gyroscopes[J]. Micromachines, 2021, 12(5).

[5] Pagani, L.G.; Dellea, S.; Bursi, G.; Brunetto, M.; Langfeilder, G. Enhancing Vibration Robustness and Noise in Automotive Gyroscope with Large Drive Motion and Levered Sense Mode[C]// 2019 IEEE 32nd International Conference on Micro Electro Mechanical Systems (MEMS). IEEE, 2019.

[6] Prandi.; Caminada.; Coronato.; Cazzaniga.; Antonello. A low-power 3-axis digital-output MEMS gyroscope with single drive and multiplexed angular rate readout[C]// Solid-state Circuits Conference Digest of Technical Papers. IEEE, 2011.

[7] Guo, Z.;Fu, P.; Liu, D.; Huang, M. Design and FEM simulation for a novel resonant silicon MEMS gyroscope with temperature compensation function[J]. Microsystem Technologies, 2017.

[8] Cui, M.; Y,Huang.; Wang, W.; Cao, H. MEMS Gyroscope Temperature Compensation Based on Drive Mode Vibration Characteristic Control[J]. Micromachines, 2019, 10(4).

[9] Zhao, Y.; Jian, Z.; Xi, W.; Xia, G.M.; Shi, Q.; Ping, Q.A.; Ping, X.Y. A Sub-0.1\%/h Bias-Instability Split-Mode MEMS Gyroscope With CMOS Readout Circuit[J]. IEEE Journal of Solid State Circuits, 2018:1-15.

[10] Ezekwe, C.D.; Gei.ger, W.; Ohms, T. 27.3 A 3-axis open-loop gyroscope with demodulation phase error correction[C]// 2015 IEEE International Solid-State Circuits Conference - (ISSCC) Digest of Technical Papers. IEEE, 2015.

[11] Feng, Bu.; Shu.wen, Guo.; Meng.meng, Cheng.; Fen, Zheng.; Da.cheng, Xu.; He.ming, Zhao. Effect of circuit phase delay on bias stability of MEMS gyroscope under force rebalance detection and self-compensation method[J]. Journal of Micromechanics \& Microengineering, 2019, 29(9):95002-95002. 
[12] Qiang, Shen.; Hong.long, Chang.; Yi.xuan, Wu.; Jian.bin, Xie. Turn-on bias behavior prediction for micromachined Coriolis vibratory gyroscopes[J]. Measurement, 2019.

[13] Yilmaz,E.; Bindel, D. Temperature Sensitivity and Shape Optimization of Solid-State Wave Gyroscopes[J]. IEEE Sensors Journal, 2016, 16(16):1-1.

[14] Hao, Y.; Yuan, W.; Xie, J.; Qiang, S.; Chang, H. Design and Verification of a Structure for Isolating Packaging Stress in SOI MEMS Devices[J]. IEEE Sensors Journal, 2016, PP(99):1-1.

[15] Zhang, M.; Yang, J.; He, Y.; Yang, F.; Yang, F.; G, Han.; C, Si.; Ning, J. Research on a 3D Encapsulation Technique for Capaci-tive MEMS Sensors Based on Through Silicon Via[J]. Sensors, 2018, 19(1).

[16] Marx, M.; Dorigo, D.D.; Nessler, S.; Rombach, S.; Manoli, Y. 9.4 A 27 $\mu$ W 0.06mm 2 background resonance frequency tuning circuit based on noise observation for a $1.71 \mathrm{~mW}$ CT- $\Delta \Sigma$ MEMS gyroscope readout system with $0.9^{\circ} / \mathrm{h}$ bias instability[C]// Solid-state Circuits Conference. IEEE, 2017.

[17] Weinberg, M.S. How to invent (or not invent) the first silicon MEMS gyroscope. IEEE, 2015.

[18] Vig, J.R. Temperature-insensitive dual-mode resonant sensors - a review[J]. IEEE Sensors Journal, 2002, 1(1):62-68.

[19] Zhao, Y.; Jian, Z.; Xi, W.; Xia, G.M.; Shi, Q.; Ping, Q.A.; Ping, X.Y. A Sub-0.1\%/h Bias-Instability Split-Mode MEMS Gyroscope With CMOS Readout Circuit[J]. IEEE Journal of Solid State Circuits, 2018:1-15.

[20] Choa, S.H. Reliability of MEMS packaging: vacuum maintenance and packaging induced stress[J]. Microsystem Technologies, 2005, 11(11):1187-1196.

[21] Li, G.; Tseng, A.A. Low stress packaging of a micromachined accelerometer[J]. IEEE Transactions on Electronics Packaging Manufacturing, 2001, 24(1):18-25. 